\title{
Association of BMP6 Methylation and Expression with Clinicopathological Features in Colorectal Cancer
}

\author{
P. Sangplod, S. Sangkhathat, T. Boonpipattanapong, and S. Kanngurn
}

\begin{abstract}
Purpose: Bone morphogenetic protein 6 (BMP6) is a member of the transforming growth factor-beta (TGF- $\beta$ ) superfamily known to regulate cell proliferation, differentiation and apoptosis. Promoter methylation of BMP6 has been reported in hematopoietic neoplasm and influences carcinogenesis and tumor progression. In the present study, we evaluated the methylation status and expression of BMP6 in colorectal cancer. Methods: A methylation-specific polymerase chain reaction was used to evaluate the methylation status of BMP6. Immunohistochemistry was used to determine the BMP6 protein expression. A total of 68 colorectal cancers $(n=68)$ were included in this analysis. Results: The methylation study of BMP6 revealed hypermethylation status in 30 cases $(44 \%)$. Promoter hypermethylation of BMP6 was significantly associated with decreased protein expression. Conclusion: Our findings suggest that BMP6 is potentially a methylationsilenced tumor suppressor gene for colorectal cancer.
\end{abstract}

Index Terms-Bone morphogenetic protein 6 (BMP6), DNA methylation, colorectal cancer.

\section{INTRODUCTION}

Colorectal cancer (CRC) is the third most commonly diagnosed cancer in males and the second in females, with over 1.2 million new cancer cases and 608,700 deaths estimated to have occurred in 2008 [1], [2]. CRC death rates have been increasing in Thailand where CRC is the third most common malignancy in males after hepatobiliary and lung cancers, and the fifth in female after cancers of the cervix, breast, hepatobiliary and lung. The number of colorectal cancer cases in both sexes is increasing and will probably exceed that of lung cancer in the next decade [3]. CRC is one of the cancers that can be prevented by secondary prevention. The precursor of advanced colorectal cancer is either an adenomatous polyp or a flat neoplastic lesion. The majority of cancers arising in the colon and rectum is adenocarcinoma that accounts for more than $90 \%$ of all large bowel tumors. However, it can be cured by the detection at an early stage and even prevented by the removal of adenomas [3].

Manuscript received January 10, 2013; revised March 15, 2013.

P. Sangplod is with Department of Biomedical Science, Faculty of Medicine, Prince of Songkla University, Songkhla, Thailand.

S. Sangkhathat is with Department of Biomedical Science and Department of Surgery and Tumor Biology Research Unit, Faculty of Medicine, Prince of Songkla University, Songkhla, Thailand (e-mail: surasak.sa@psu.ac.th).

T. Boonpipattanapong is with Department of Surgery, Faculty of Medicine, Prince of Songkla University, Songkhla, Thailand.

S. Kanngurn is with Tumor Biology Research Unit and Department of Pathology, Faculty of Medicine, Prince of Songkla University, Songkhla, Thailand.
Bone morphogenetic proteins (BMPs) belong to the transforming growth factor- $\beta$ superfamily. They are multifunctional cytokines that control cellular responses including the induction of cell growth inhibition, differentiation and apoptosis [4]. BMP6 is an autocrine stimulator of chondrocyte differentiation [5] and has been implicated in the development of embryonic kidney and urinary systems [6]. Moreover, BMP6 expression can be localized to muscle cells in the developing human fetal intestine [7]. In vitro, BMP6 has been shown to inhibit cell division, to promote terminal epithelial differentiation, and to induce endochondral bone formation, osteoblastic differentiation, and neuronal maturation [8]. Furthermore, in prostatic adenocarcinoma, induction of BMP6 expression has been associated with tumorigenesis [9] and the formation of osteosclerotic deposits in metastatic progression [10]. In addition, it has been reported that BMP6 may play an important role in heterotopic ossification in colon adenocarcinoma. It is prominent in the cytoplasm of tumor cells, and it stains weakly in osteoblast-like cells adjacent to newly formed bone [11]. In addition, several reports suggested that BMP6 promoter methylation status is correlated with cancer.

Recently, promoter methylation of the BMP6 gene has been shown in aggressive types of cancer for example, hypermethylation of BMP6 is common in human prostate cancer [12], and it plays a role in breast cancer development and metastasis [13]. However, data on the association of BMP6 in CRC is not clear. Therefore, our study will examine the association of the DNA methylation and its protein expression of BMP6 in CRC. Finally, we hope that BMP6 will be used in the diagnosis in patients with CRC.

\section{MATERIALS AND METHODS}

Sample collection: A total of 68 cases from patients who had surgery in Songklanagarind Hospital from 2008 to 2010 were included in this study. Fresh tissues and formalin-fixed paraffin embedded (FFPE) CRC tissue samples were used to study BMP6 methylation and expression, respectively.

Methylation specific polymerase chain reaction (MSP): MSP was used to observe the BMP6 promotor methylation in CRC. DNA from fresh tissue was extracted using QIAamp DNA Mini Kit (Qiagen) according to the enclosed protocol. DNA was modified by bisulfite treatment using EZ DNA Methylation ${ }^{\mathrm{TM}}$ Kit (Zymo Research Corp.). The primers were 5'-TTGGGTAGTTG GGTGATTGTT-3' (sense) and 5'-ACACCCCTCCCCAA ATCA-3' (antisense) for un- methylated form and 5'GGTTTGTTGGGTAGTCGGG-3' (sense) and 5'-GCCCC 
TCCCCAAATCG-3' (antisense) for the methylated form which give a PCR product of $248 \mathrm{bp}$, encompassing the BMP6 promoter region -836 to -589 bp relative to the transcriptional start site. Two pairs of primer, methylated and unmethylated, were used to amplify by HotStar Taq (Qiagen). The PCR was performed under the following conditions: $95^{\circ} \mathrm{C} 15 \mathrm{~min}$, followed by 39 cycles at $94^{\circ} \mathrm{C}$ for $30 \mathrm{~s}, 64.4^{\circ} \mathrm{C}$ for $30 \mathrm{~s}$ and $72^{\circ} \mathrm{C}$ for $45 \mathrm{~s}$. Human methylated $\&$ non-methylated DNA sets (Zymo Research Corp.) were used as negative and positive controls, respectively. Finally, PCR products were detected by $2 \%$ agarose gel electrophoresis and stained with ethidium bromide.

Immunohistochemistry (IHC): IHC was used to study the expression of BMP6 protein in CRC. FFPE tissues placed on slides were deparaffinized by heating for $30 \mathrm{~min}$ at $60^{\circ} \mathrm{C}$, followed by soaking them twice in xylene, followed by rehydration in a series of graded alcohols. The slides were immersed in $1 \mathrm{X}$ Tris EDTA buffer ( $\mathrm{pH} 9.0)$ and heated in a microwave for $10 \mathrm{~min}$ for antigen retrieval.

Endogenous peroxidase activity was blocked by incubation with $3 \% \mathrm{H}_{2} \mathrm{O}_{2}$. Horse serum $(10 \%)$ was added for 30 min to block nonspecific protein binding. The slides were incubated with mouse monoclonal anti-BMP6 antibody (Abcam plc) which was used as the primary antibody at a concentration of a 1:100 dilutions for overnight at $4{ }^{\circ} \mathrm{C}$. BMP6 protein was detected by the EnVision ${ }^{\mathrm{TM}}+$ system (DAKO) according to the manufacturer's instructions. Finally, the slides were incubated with 3, 3'diaminobenzidine (Sigma-Aldrich) until a brown color developed, and counterstained with Harris' modified hematoxylin. In negative controls, primary antibody was omitted. For the evaluation of BMP6 expression, slides of cancer specimens were analyzed in parallel by two investigators in an effort to provide a consensus on staining patterns by light microscopy. The Allred scoring system was the standard scoring system used to evaluate stain intensity and stain pattern. The numerical value for overall intensity (intensity score, IS) was based on a 4 point system: $0,1,2$, and 3 (for none, light, medium, or dark staining). The numerical value for percent stained (proportion score, PS) was determined by a geometric rather than linear division no staining $=0 ; \leq 1 / 100$ cells stained $=1 ; \leq 1 / 10$ cells stained $=$ $2 ; \leq 1 / 3$ cells stained $=3, \leq 2 / 3$ cells stained $=4$; all cells stained $=5$. Addition of the two values gave the total Allred score, so the Allred score could vary between 0 and 8 [14].

\section{StATISTICS}

For clinicopathological parameters, data is presented as mean or percent. The association between the BMP6 methylation status and immunohistochemical score was determined by using the Chi-square test. $P$ values $<0.05$ were considered statistically significant.

\section{RESULTS}

A total of 68 CRC (36 females and 32 males) were included in this analysis. The mean age (range) of the patients was 64 years (36-87 years) with 39 cases $(57 \%)$ aged more than 60 years. Regarding the sites of the primary tumor, 42 cases $(63 \%)$ were rectal in origin and 24 cases were colonic cancer., Approximately $65 \%$ of the cases were categorized as stage 3-4 on diagnostic work-up (Table I).

The methylation study of BMP6 revealed hypermethylation status in 30 cases $(44 \%)$. There was no statistically significant association between BMP6 hypermethylation and clinicopathological parameters of the CRCs studied (Table I).

TABLE I: Clinicopatological Data and BMP6 Methylation Status of 68 Colorectal CANCER CASES Studied

\begin{tabular}{|c|c|c|c|c|c|}
\hline \multirow[b]{2}{*}{ Parameter } & & \multicolumn{4}{|c|}{ Methylation status } \\
\hline & & $\mathrm{N}$ (cases)* & negative & positive & $\mathrm{p}$-value \\
\hline All & & 68 & $38(56 \%)$ & $30(44 \%)$ & \\
\hline \multicolumn{6}{|l|}{ Sex } \\
\hline & female & 36 & $23(64 \%)$ & $13(36 \%)$ & 0.16 \\
\hline & male & 32 & $15(47 \%)$ & $17(53 \%)$ & \\
\hline \multicolumn{6}{|l|}{ Age } \\
\hline & $0-60$ years & 29 & $16(55 \%)$ & $13(45 \%)$ & 0.92 \\
\hline & $>60$ years & 39 & $22(56 \%)$ & $17(44 \%)$ & \\
\hline \multicolumn{6}{|c|}{ Site of primary tumor } \\
\hline & Rectum & 42 & $25(60 \%)$ & $17(40 \%)$ & 0.67 \\
\hline & Colon & 24 & $13(54 \%)$ & $11(46 \%)$ & \\
\hline \multicolumn{6}{|c|}{ AJCC stage } \\
\hline & Stage $1-2$ & 23 & $12(52 \%)$ & $11(48 \%)$ & 0.52 \\
\hline & Stage 3-4 & 43 & $26(60 \%)$ & $17(40 \%)$ & \\
\hline \multicolumn{6}{|c|}{ Nodal status } \\
\hline & No & 24 & $12(50 \%)$ & $12(50 \%)$ & 0.30 \\
\hline & N1 & 21 & $11(52 \%)$ & $10(48 \%)$ & \\
\hline & $\mathrm{N} 2$ & 21 & $15(71 \%)$ & $6(29 \%)$ & \\
\hline \multicolumn{6}{|c|}{ Differentiation } \\
\hline & Well & 28 & $19(68 \%)$ & $9(32 \%)$ & 0.159 \\
\hline & $\begin{array}{r}\text { Moderate } \\
\text { Poor }\end{array}$ & $\begin{array}{c}27 \\
7\end{array}$ & $\begin{array}{c}15(56 \%) \\
2(29 \%)\end{array}$ & $\begin{array}{c}12(44 \%) \\
5(71 \%)\end{array}$ & \\
\hline
\end{tabular}




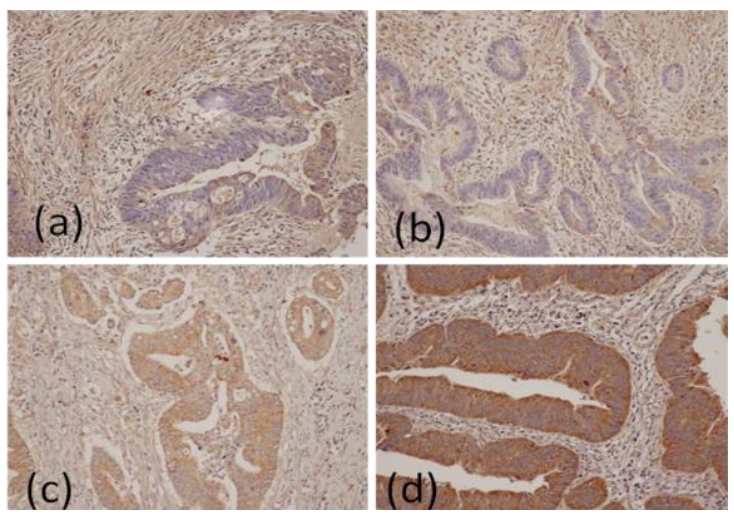

Fig. 1. Representative pictures of immunohistochemistry on CRC specimens stained for BMP6 antibody. (a) and (b) do not show no expression of BMP6 in tumor cells. (c) and (d) show expression of BMP6 in tumor cells. Magnification: X20.

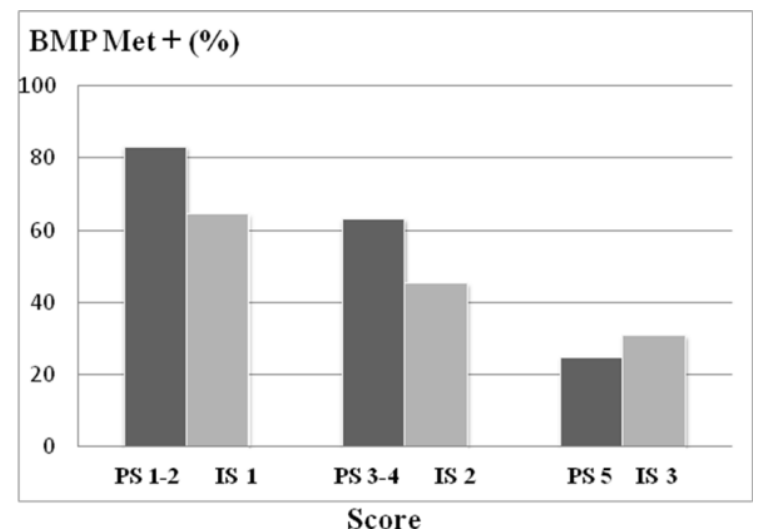

Fig. 2. Percentage of BMP6 hypermethylation according to proportion score (PS) and intensity score (IS); $p$-value 0.02

On immunohistochemical study, positive BMP6 immunoreactivity could be detected in both tumor cells and stroma (Fig. 1). The average proportion score (PS) was 4.0. The PS showed a significant inverse correlation with hypermethylation status. (Fig. 2)

The average intensity score (IS) was 2.17 . Consistent with the PS, the IS also showed an inverse correlation with the BMP6 hypermethylation rate. The IS of 1,2 and 3 had an incidence of hypermethylation at $65 \%, 45 \%$ and $31 \%$, respectively. However, the correlation did not reach a statistically significant level. When PS and IS were taken together as the Allred score (AS), hypermethylated tumor had a significantly lower AS (Fig. 3). Serial analysis revealed that a cut-off value at 4 provided the lowest $\mathrm{p}$-value on the Chi-square test $(p$-value $=0.006)$. Tumors with an AS of 4 or less had an $82 \%$ incidence of hypermethylation when $37 \%$ of tumor with higher score had the status.

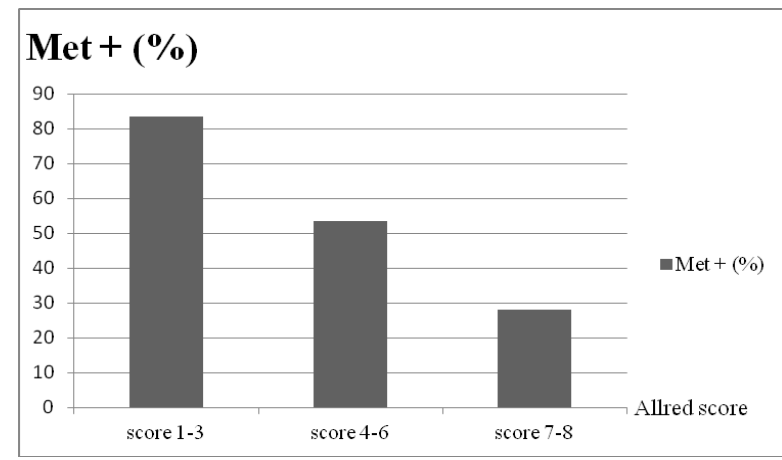

Fig. 3. Percentage of BMP6 hypermethylation according to allred score (sum of proportion score and intensity score); $p$-value 0.02 .

\section{DISCUSSION}

BMP is a member of the TGF- $\beta$ superfamily of signaling molecules which are important to inhibit cellular proliferation and is involved in organogenesis, particularly of the lung, heart and kidney [15], [16]. BMP6 is similar to other BMP members which signals through ligation of type I and type II serine-threonine kinase receptors (BMPR) and signal downstream by phosphorylation of Smad1, 5, and 8. These Smads then form complexes with the Smad4 and are translocated into a nucleus where they exert regulation of target genes specific for the BMP pathway. Thus, BMP signaling is similar to the paradigm established by TGF- $\beta$ signaling. It is logical, therefore, to suppose that any functional impairment by genetic alterations or epigenetic inactivation of genes involved in the BMP/TGF- $\beta$ pathway may induce the development of cancers. There are many reports that described genetic alterations or epigenetic inactivation of BMP6 which was shown to be associated with tumorigenesis and/or disease progression in several cancers [17]. BMP6 inhibited proliferation of prostate cancer cells by up-regulation of several cyclin-dependent kinase inhibitors. Loss of sensitivity to BMP6 is necessary to achieve the malignant phenotypes [18]-[20]. Furthermore, epigenetic inactivation of BMP6 by gene promoter hypermethylation promoted lung tumor development [21].

In this study, we have analyzed the methylation status of the BMP6 promoter region in CRC samples. We found intensive promoter methylation with significant high frequency in CRC which suggests that the BMP6 promoter methylation seems to be tumor specific. On the other hand, the BMP6 methylation status and the clinicopathology were not associated with statistical significance. We also showed methylation-dependent loss of BMP6 expression protein levels. In fact, methylation was observed to play a role in mediating gene expression. Evidence of this has been found in other studies that show that methylation near gene promoters varies considerably depending on cell type, with more methylation of promoters correlating with low or no transcription of the gene. Thus, our findings implied a causal relationship between methylation of the BMP6 promoter and transcriptional repression. Our data are, to the best of our knowledge, the first demonstration of epigenetic inactivation of a BMP family member in CRC.

In summary, our study showed for the first time that the promoter of the BMP6 gene was methylated more often in CRC. This study also supports the role of BMP6 with tumor suppressor genes in CRC. Furthermore, the BMP6 promoter methylation profile seems to be an important marker in predicting the clinical outcome in CRC. An understanding of the BMP signaling pathways in $\mathrm{CRC}$ will be valuable in elucidating the molecular mechanisms in the roles of BMPs in CRC and novel strategies for their treatment.

\section{REFERENCES}

[1] B. W. Stewart and P. Kleihues, World Cancer Report, Lyon: IARC Press, 2003, pp. 347-380.

[2] A. Jemal, F. Bray, M. M. Center, J. Ferlay, E. Ward, and D. Forman, "Global Cancer Statistics," Cancer J Clin, vol. 61, no. 2, pp. 69-90, 2011.

[3] T. Khuhaprema and P. Srivatanakul, "Colon and rectum cancer in Thailand: An overview," Oncol, vol. 38, no. 4, pp. 237-243, 2008.

[4] A. Taniguchi, Y. Nemoto, A. Yokoyama, N. Kotani, T. Shuin, and M. Daibata, "Promoter methylation of the bone morphogenetic protein-6 
gene in association with adult T-Cell Leukemia," Int J Cancer, vol. 123 , no. 8, pp. 1824-1831, 2008.

[5] C. D. Grimsrud et al., "BMP-6 is an autocrine stimulator of chondrocyte differentiation," J Bone Miner Res, vol. 14, no. 4, pp. 475-482, 1999.

[6] M. Hebrok, S. K. Kim, B. S. Jacques, A. P. McMahon, and D. A. Melton, "Regulation of pancreas development by hedgehog signaling," Development, vol. 127, no. 22, pp. 4905-4913, 2000.

[7] H. A. Perr, J. Ye, and S. E. Gitelman, "Smooth muscle expresses bone morphogenetic protein (Vgr-1/BMP-6) in human fetal intestine," Biol Neonate, vol. 75, no. 3, pp. 210-214, 1999.

[8] V. Drozdoff, N. A. Wall, and W.J. Pledge, "Expression and growth inhibitory effect of decapentaplegic-Vg-related protein 6: evidence for a regulatory role in keratinocyte differentiation,"in Proc Natl Acad Sci USA, vol. 91, no. 12, 1994, pp. 5528-5532.

[9] F. C. Hamdy, P. Autzen, M. C. Robinson, C. H. W. Home, D. E. Neal, and C. N. Robson, "Immunolocalization and messenger RNA expression of Bone Morphogenetic Protein- 6 in human benign and malignant prostatic tissue," Cancer Res, vol. 19, no. 57, pp. 44274431, 1997.

[10] H. Bentley et al., "Expression of bone morphogenetic proteins in human prostatic adenocarcinoma and benign prostatic hyperplasia," Br J Cancer, vol. 66, no. 6, pp. 1159-1163, 1992.

[11] N. Ima et al., "Expression of bone morphogenetic proteins in colon carcinoma with heterotopic ossification," Pathol Int, vol. 51, no. 8, pp 643-648, 2001

[12] H. Tamada, R. Kitazawa, K. Gohji, and S. Kitazawa, "Epigenetic regulation of human Bone Morphogenetic Protein 6 gene expression in prostate cancer," J Bone Miner Res, vol. 16, no. 3, pp. 487-495, 2001.

[13] M. Zhang et al., "Epigenetic regulation of bone morphogenetic protein-6 gene expression in breast cancer cells," J Steroid Biochemistry Mol Biol, vol. 105, no. 1-5, pp. 91-97, 2007

[14] K. R. Choudhury, K. J. Yagle, P. E. Swanson, K. A. Krohn, and J. G. Rajendran, "A robust automated measure of average antibody staining in immunohistochemistry images," J Histochem Cytochem, vol. 58, no. 2, pp. 95-107, 2010.

[15] K. Kimura et al., "The aberrant promoter methylation of BMP3b and BMP6 in malignant pleural mesotheliomas," Oncol Rep, vol. 20, no. 5, pp. $1265-1268,2008$

[16] J. Du et al., "BMP-6 inhibits microRNA-21 expression in breast cancer through repressing deltaEF1 and AP-1," Cell Res, vol. 19, no. 4, pp. 487-496, 2009.

[17] T. B. Ro et al., "Bone morphogenetic protein-5, -6 and -7 inhibit growth and induce apoptosis in human myeloma cells," Oncogene, vol. 23, no. 17, pp. 3024-3032, 2004

[18] Y. T. Xiao, L. X. Xiang, and J. Z. Shao, "Bone morphogenetic protein," Biochem Biophys Res Commun, vol. 362, no. 3, pp. 550-553, 2007.

[19] J. W. Lowery and M. P. de Caestecker, "BMP signaling in vascular development and disease," Cytokine and growth Factor Reviews, vol. 21, no. 6, pp. 287-298, 2010.

[20] I. Y. Kim et al., "Decreasedexpression of bone morphogenetic protein (BMP) receptor type II correlates with insensitivity to BMP-6 in human renal cell carcinoma cells," Clinical Cancer Res, vol. 9, no. 16, pp. 6046-6051, 2003

[21] K. S. Kraunz, H. H. Nelson, M. Liu, J. K. Wiencke, and K. T. Kelsey, "Interaction between the bone morphogenetic proteins and Ras/MAPkinase signaling pathways in lung cancer," Br J Cancer, vol. 93, no. 8, pp. 949-952, 2005 .

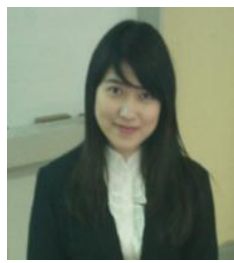

Patcharaporn Sangplod obtained her B.Sc degree. She is currently a master degree student in the Program of Biomedical Science, Department of Biomedical Science, Faculty of Medicine, Prince of Songkhla University, Thailand. She also has an education background in chemistry from the Faculty of Science, Prince of Songkla University. Her research interest is molecular pathogenesis of colorectal cancers, focusing on methylation of tumor suppressor genes.

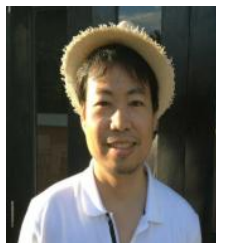

Surasak Sangkhathat obtained his M.D. and Ph.D. $\mathrm{He}$ is a pediatric surgeon who has his extended interest in the molecular biology of cancer, especially colorectal cancer. He is currently a Chairperson of Tumor Biology Research Unit, Prince of Songkla University.

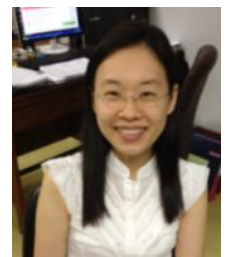

Teeranut Boonpipattanapong obtained her M.D. degree. She is a renowned colorectal surgeon practicing in the Department of Surgery, Songklanagarind Hospital and a member of the Tumor Biology Research Unit. She is recognized among patients and students as a hard-working surgeon and medical teacher. Her special area of interest is at outcome studies in colorectal cancer.

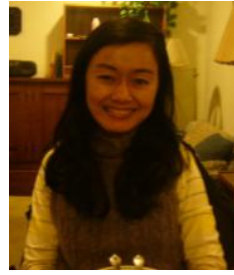

Samornmas Kanngern obtained her M.D. degree. She is a pathologist who devoted herself for gastrointestinal pathology. Her works are focusing in molecular biomarkers in colorectal cancers, gastrointestinal stromal tumor and cholangiocarcinoma. 